\title{
CAPACIDADE REPRODUTIVA E LONGEVIDADE DE Chrysoperla externa CUJAS FASES IMATURAS FORAM SUBMETIDAS À AMBIENTES COM DIFERENTES TEMPERATURAS DE CRIAÇÃO
}

\author{
LAVAGNINI, Taís Carmona ${ }^{1}$
}

FREITAS, Sérgio ${ }^{1}$

\begin{abstract}
RESUMO: Os crisopídeos ocorrem em diversos agroecossistemas e alimentam-se de diversos artrópodes-praga e, portanto podem ser utilizados como agentes em programas de controle biológico de pragas agrícolas. O sucesso dos crisopídeos pode estar relacionado a sua capacidade de sobrevivência e reprodução em campo. Esta característica pode ser alterada por diversos fatores, inclusive a temperatura. Objetivou-se com este estudo verificar se as temperaturas de criação das fases imaturas de Chrysoperla externa (Hagen, 1861) podem influenciar a sua capacidade reprodutiva e a longevidade dos adultos. As fases imaturas foram criadas em quatro ambientes de criação, três com temperaturas constantes de 19,25 e $31^{\circ} \mathrm{C}$ e outro ambiente em que houve flutuação dos fatores abióticos. Os adultos obtidos foram mantidos em salas climatizadas à $25^{\circ} \mathrm{C}$ para avaliação da capacidade reprodutiva e da longevidade. Os dados obtidos não diferiram significativamente entre os ambientes onde as fases imaturas foram criadas. Conclui-se que a capacidade reprodutiva e a longevidade não serão alteradas quando as fases jovens forem criadas em temperaturas diferentes.
\end{abstract}

Palavras-chave: Biologia. Chrysoperla externa. Criação. Crisopídeos. Reprodução.

\section{REPRODUCTIVE CAPACITY AND LONGEVITY OF Chrysoperla externa WHOSE IMMATURE STAGES WERE SUBMITTED TO DIFFERENT REARING TEMPERATURES}

SUMMARY: The green lacewings occur in many agroecosystems and eat a lot of pests, so they can be used in biological control programs for agricultural pests. The success of them is related with their capacity of living in this kind of environment and reproduce. This attribute can be influenced for many factors, such as temperature. The purpose of this study was to check if different rearing temperatures of immature stages of Chrysoperla externa (Hagen, 1861) can influence their reproductive capacity and longevity. The immature stages were reared in four environments, three of them with constants temperatures of 19,25 and $31^{\circ} \mathrm{C}$ and in the fourth environment the abiotic factors were not controlled. The adults obtained of this rearing were maintained at $25^{\circ} \mathrm{C}$ for evaluation of reproductive capacity and longevity. The results obtained didn't differ significantly among the environments where the immature stages were kept. It can be concluded that the reproductive capacity and longevity will not influenced when the immature stages were reared in different temperatures.

Keywords: Biology. Chrysoperla externa. Green lacewings. Rearing. Reproduction.

\section{INTRODUÇÃO}

Os crisopídeos ocorrem em diversos agroecossistemas destacando-se como eficazes predadores de artrópodes-praga, controlando assim a densidade populacional destes organismos. Sua utilização em programas de controle biológico se deve ao fato de serem facilmente criados em laboratório, possuírem alto potencial reprodutivo, serem vorazes predadores, além de apresentarem tolerância a determinados grupos de inseticidas (MAIA et al., 2004).

Os limites da faixa favorável de temperatura para oviposição são geralmente os da mesma ordem para o desenvolvimento de estágios imaturos, mas não precisam ser necessariamente similares. Contudo, outros fatores além da temperatura podem influenciar a oviposição como umidade relativa, alimento e o número de indivíduos presentes no ambiente; o número de ovos por dia pode ser influenciado por estes

\footnotetext{
${ }^{1}$ UNESP, Campus Jaboticabal - SP. Departamento de Fitossanidade.
} 
fatores e também pela idade do indivíduo (ANDREWARTHA; BIRCH, 1954).

O período de pré-oviposição em crisopídeos, que não apresentam diapausa, estende-se entre 4 a 10 dias em condições ótimas (CANARD; VOLKOVICH, 2001). Estudos realizados com fêmeas adultas de Chrysoperla externa (Hagen, 1861) mantidas a $21,1^{\circ} \mathrm{C}$ mostraram que o período de pré-oviposição durou aproximadamente 10 dias (ALBUQUERQUE et al., 1994). Quanto à longevidade, Núñez (1988) observou que a $25^{\circ} \mathrm{C}$ a longevidade dos machos de C. externa era de aproximadamente 31 dias e das fêmeas 49 dias. A idade das fêmeas pode influenciar a capacidade de oviposição, uma vez que o número de ovos colocados por fêmeas de C. externa aumenta gradativamente alcançando seu pico no $49^{\circ}$ dia. A partir daí, ocorre um acentuado decréscimo na capacidade de oviposição sendo que a menor quantidade de ovos é observada aos 63 dias de idade (MACEDO et al., 2003b).

Determinados fatores abióticos, entre eles a temperatura, podem influenciar o desenvolvimento de ovos e larvas, pois alguns processos fisiológicos sofrem alterações de acordo com a temperatura do ambiente. Desta forma, o objetivo deste trabalho foi verificar se tais alterações fisiológicas geradas nas fases imaturas de $C$. externa, criadas em diferentes temperaturas, pode influenciar a capacidade reprodutiva e a longevidade dos adultos.

\section{MATERIAL E MÉTODOS}

Crisopídeos adultos foram coletados com auxílio de rede entomológica e levados para o laboratório para identificação da espécie e para que fosse estabelecida a criação estoque para o experimento. Seus ovos (geração $F_{1}$ ) foram colocados em arenas de criação $(12 \mathrm{~cm} \mathrm{x} 8 \mathrm{~cm}$ ), 20 ovos cada, contendo tiras de papel higiênico para redução da taxa de canibalismo entre as larvas. A partir dos adultos da geração $F_{1}$ foram obtidos ovos (geração $F_{2}$ ) que foram utilizados na experimentação. Em todas as gerações as larvas foram alimentadas com ovos de Sitotroga cereallella (Oliver, 1819) (Lepidoptera: Gelechiidae) e os adultos com uma dieta a base de mel e lêvedo de cerveja na proporção 1:1.

Os ovos da geração $F_{2}$ foram individualizados em recipientes de vidro transparente e incolor com $1,5 \mathrm{~cm}$ de diâmetro e 2,5 cm de altura, vedados com filme plástico. As fases imaturas foram submetidas a diferentes temperaturas de criação. Três grupos foram conduzidos em salas climatizadas com temperaturas constantes de 19,25 e $31^{\circ} \mathrm{C}$, fotoperíodo de 12 horas e umidade relativa de $70 \pm 10 \%$. O quarto grupo foi conduzido em uma área coberta fora do laboratório para que houvesse flutuação dos fatores abióticos como temperatura, fotoperíodo e umidade relativa do ar. Em cada grupo havia 150 indivíduos.

Ao final do período pupal os adultos obtidos foram separados em gaiolas de tubo de PVC $(20 \mathrm{x} 10$ $\mathrm{cm})$ revestidas internamente com papel sulfite. Foi colocado um casal por gaiola e todos os grupos foram mantidos em uma sala climatizada com temperatura constante de $25 \pm 2^{\circ} \mathrm{C}$, fotoperíodo de 12 horas e umidade relativa de $70 \pm 10 \%$. Em virtude das diferentes taxas de emergência observadas para os indivíduos criados em cada ambiente, foram avaliados números diferentes de casais em cada tratamento. Para os ambientes em que as fases imaturas foram criadas à 19,25 e $31^{\circ} \mathrm{C}$ e no ambiente com flutuação dos fatores abióticos foram avaliados 12, 12, 10 e 20 casais, respectivamente. Desta forma, cada casal foi considerado com uma repetição dentro do seu tratamento.

Diariamente os ovos foram retirados, contados e, uma vez por semana, foi avaliada a porcentagem de viabilidade dos ovos. Para esta avaliação os ovos foram individualizados em placas de teste ELISA cobertas com filme plástico. Depois de dois dias os ovos foram observados para determinação da porcentagem de ovos viáveis.

Foram observados a duração do período de pré-oviposição, oviposição e pós-oviposição, longevidade de machos e fêmeas, número de ovos por dia e total por fêmea e a viabilidade dos ovos. 
Os dados obtidos foram submetidos à análise de variância pelo teste $\mathrm{F}$ e as médias foram comparadas por meio do Teste de Tukey $(\mathrm{p}<0,05)$. Apenas os dados referentes ao número total de ovos por fêmea foram transformados segundo $\sqrt{x+1}$. As análises foram realizadas através do software Estat (ESTAT, 1992).

\section{RESULTADOS E DISCUSSÃO}

Os fatores abióticos como temperatura e umidade relativa, para o ambiente em que houve flutuação dos mesmos, foram registrados com o auxílio de um termohigrômetro. A temperatura média obtida foi de $24 \pm 0,8^{\circ} \mathrm{C}$, sendo a máxima $31,7^{\circ} \mathrm{C}$ e a mínima $17,0^{\circ} \mathrm{C}$; a umidade relativa média foi de $64,3 \%$, máxima de $96,76 \%$ e mínima de $31,82 \%$; o fotoperíodo foi natural.

A baixa temperatura $\left(19^{\circ} \mathrm{C}\right)$ de criação das fases imaturas promoveu um período de pré-oviposição (Tabela 1) mais longo que nas demais fêmeas, entretanto as fêmeas cujas fases imaturas foram criadas a $24^{\circ} \mathrm{C}(\mathrm{Amb})$ apresentaram período de pré-oviposição mais curto, provavelmente devido a intensa flutuação da temperatura ao longo do dia. Ambos os resultados apresentaram diferenças significativas.

Tabela 1. Duração (dias) dos períodos de pré-oviposição, oviposição e pós-oviposição, número de total de ovos por fêmea, número de ovos/dia/fêmea, viabilidade de ovos (\%) e longevidade (dias) de machos e fêmeas de Chrysoperla externa cujos ovos e larvas foram submetidos às temperaturas de criação de $19^{\circ} \mathrm{C}$, $24^{\circ} \mathrm{C}(\mathrm{Amb}), 25^{\circ} \mathrm{C}$ e $31^{\circ} \mathrm{C}$.

\begin{tabular}{ccccc}
\hline & $\begin{array}{c}\text { Período de pré- } \\
\text { oviposição }\end{array}$ & $\begin{array}{c}\text { Período de } \\
\text { oviposição }\end{array}$ & $\begin{array}{c}\text { Período de pós- } \\
\text { oviposição }\end{array}$ & $\begin{array}{c}\text { Número total de } \\
\text { ovos/fêmea }\end{array}$ \\
\hline $\begin{array}{c}\mathbf{1 9}^{\circ} \mathbf{C} \\
(\mathbf{n = 1 2})\end{array}$ & $6,5 \pm 0,7 \mathrm{~b}$ & $46,4 \pm 3,7 \mathrm{a}$ & $4,0 \pm 0,8 \mathrm{a}$ & $770,0 \pm 106,5 \mathrm{a}$ \\
$\begin{array}{c}\mathbf{2 4}^{\circ} \mathbf{C}(\mathbf{A m b}) \\
(\mathbf{n = 2 0})\end{array}$ & $4,6 \pm 0,2 \mathrm{a}$ & $48,0 \pm 2,4 \mathrm{a}$ & $5,1 \pm 0,9 \mathrm{a}$ & $871,8 \pm 64,2 \mathrm{a}$ \\
$\begin{array}{c}\mathbf{2 5}{ }^{\circ} \mathbf{C} \\
(\mathbf{n = 1 2})\end{array}$ & $5,1 \pm 0,4 \mathrm{ab}$ & $47,0 \pm 4,7 \mathrm{a}$ & $8,0 \pm 2,1 \mathrm{a}$ & $861,5 \pm 89,9 \mathrm{a}$ \\
$\begin{array}{c}\mathbf{3 1}{ }^{\circ} \mathbf{C} \\
(\mathbf{n = 1 0})\end{array}$ & $5,5 \pm 0,2 \mathrm{ab}$ & $48,3 \pm 4,8 \mathrm{a}$ & $5,4 \pm 1,8 \mathrm{a}$ & $812,3 \pm 89,5 \mathrm{a}$ \\
$\mathbf{T e s t e} \mathbf{F}$ & $3,91^{*}$ & $0,06^{\mathrm{NS}}$ & $1,32^{\mathrm{NS}}$ & $0,38^{\mathrm{NS}}$ \\
\hline & Ovos/dia/fêmea & $\begin{array}{c}\text { Viabilidade } \\
\text { de ovos }(\%)\end{array}$ & $\begin{array}{c}\text { Longevidade de } \\
\text { machos }\end{array}$ & $\begin{array}{c}\text { Longevidade de } \\
\text { fêmeas }\end{array}$ \\
\hline $\begin{array}{c}\mathbf{1 9}^{\circ} \mathbf{C} \\
(\mathbf{n = 1 2})\end{array}$ & $17,3 \pm 2,3 \mathrm{a}$ & $93,9 \pm 0,0 \mathrm{a}$ & $55,0 \pm 8,6 \mathrm{a}$ & $68,0 \pm 3,7 \mathrm{a}$ \\
\hline $\begin{array}{c}\mathbf{2 4} \mathbf{C}^{\circ} \mathbf{C}(\mathbf{A m b}) \\
(\mathbf{n = 2 0})\end{array}$ & $18,2 \pm 1,3 \mathrm{a}$ & $99,2 \pm 0,0 \mathrm{a}$ & $58,1 \pm 7,7 \mathrm{a}$ & $69,0 \pm 2,9 \mathrm{a}$ \\
\hline $\begin{array}{c}\mathbf{2 5}{ }^{\circ} \mathbf{C} \\
(\mathbf{n = 1 2})\end{array}$ & $19,0 \pm 1,2 \mathrm{a}$ & $99,5 \pm 0,0 \mathrm{a}$ & $62,1 \pm 6,7 \mathrm{a}$ & $62,0 \pm 5,7 \mathrm{a}$ \\
\hline $\begin{array}{c}\mathbf{3 1}{ }^{\circ} \mathbf{C} \\
(\mathbf{n = 1 0})\end{array}$ & $17,5 \pm 1,9 \mathrm{a}$ & $99,2 \pm 0,0 \mathrm{a}$ & $59,9 \pm 11,5 \mathrm{a}$ & $70,5 \pm 4,7 \mathrm{a}$ \\
\hline $\mathbf{T e s t e ~} \mathbf{F}$ & $0,20 \mathrm{NS}$ & $1,80 \mathrm{NS}$ & $0,17 \mathrm{NS}$ & $0,49 \mathrm{NS}$ \\
\hline
\end{tabular}

Médias \pm erro padrão seguidas pela mesma letra na coluna não são significativamente diferentes para o Teste de Tukey (P<0,05). ${ }^{1}$ Comparação feita com transformação segundo $\sqrt{x+1}$

*valores médios significativos com $\mathrm{P}<0,05$

n: número de casais avaliados

NS: valores médios não significativos

Os valores médios obtidos para os períodos de oviposição e pós-oviposição nos diferentes tratamentos não apresentaram diferença significativa entre si (Tabela 1). O número de ovos total e diário por fêmea e a viabilidade dos ovos (Tabela 1) também não diferiram significativamente entre os ambientes 
de criação de suas fases imaturas. Macedo et al. (2003b) também observaram que a capacidade de oviposição das fêmeas cujo desenvolvimento das fases imaturas ocorreu em casa de vegetação, com flutuação da temperatura $\left(18-24^{\circ} \mathrm{C}\right)$, não foi afetada pela temperatura. Desta forma, tais parâmetros biológicos parecem não ser influenciados quando os ovos e larvas de C. externa são submetidos a diferentes temperaturas de criação.

Apesar de não terem sido observadas diferenças significativas quanto ao número de ovos entre os diferentes tratamentos, pode-se notar que há diferenças entre a flutuação no número de ovos por semana de acordo com a idade das fêmeas (Figura 1). Para as fêmeas obtidas a partir de ovos e larvas criados a $19^{\circ} \mathrm{C}$ observa-se dois picos de oviposição, o primeiro pico ocorre durante a $2^{\mathrm{a}}$ semana e o segundo pico durante a $4^{\mathrm{a}}$ semana, sendo que entre eles há um declínio, durante a $3^{\mathrm{a}}$ semana. Esta redução no número de ovos seguido de um novo pico pode ser em decorrência de uma segunda cópula em função do esgotamento das reservas espermáticas (RIBEIRO; CARVALHO, 1991). Na situação em que as fases imaturas foram criadas a $25^{\circ} \mathrm{C}$, as fêmeas não apresentaram picos de oviposição bem definidos como ocorreu a $19^{\circ} \mathrm{C}$. Houve aumento gradual no número de ovos por semana, que se manteve estável por alguns dias, entre a $3^{\mathrm{a}}$ e $4^{\mathrm{a}}$ semanas, apresentando redução gradual até o encerramento do período de oviposição. Para as fêmeas cujas fases jovens foram submetidas ao ambiente com $31^{\circ} \mathrm{C}$ pode-se observar uma situação semelhante ao que ocorreu no ambiente a $25^{\circ} \mathrm{C}$, em que houve aumento e redução gradual no número de ovos por semana, mas sem que houvesse um período estável. Já para as fêmeas obtidas de ovos e larvas criados no ambiente em que houve flutuação da temperatura observou-se redução abrupta no número de ovos entre a $1^{\mathrm{a}}$ e a $2^{\mathrm{a}}$ semana, seguido por um aumento gradual que atingiu o número máximo de ovos na $4^{\mathrm{a}}$ semana, declinando até o encerramento do período de oviposição.

Figura 1. Número médio de ovos por semana para Chrysoperla externa cujas fases imaturas foram submetidas a ambientes com diferentes temperaturas de criação. Foram inseridas barras de erro-padrão para cada um dos valores médios indicados nos gráficos. a: $19^{\circ} \mathrm{C}, \mathrm{b}: 25^{\circ} \mathrm{C}, \mathrm{c}: 31^{\circ} \mathrm{C}$, d: ambiente com flutuação dos fatores abióticos.

(a)

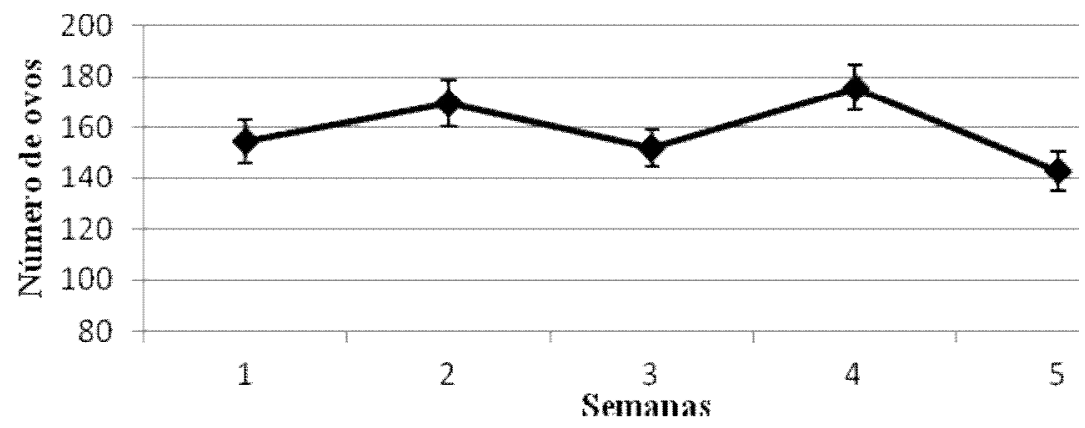

(b)

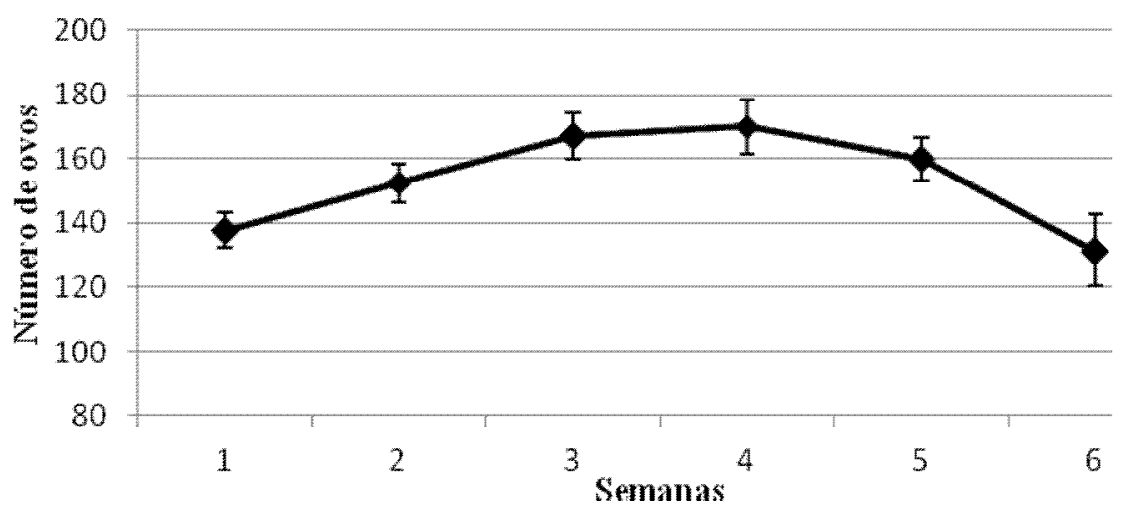


(c)
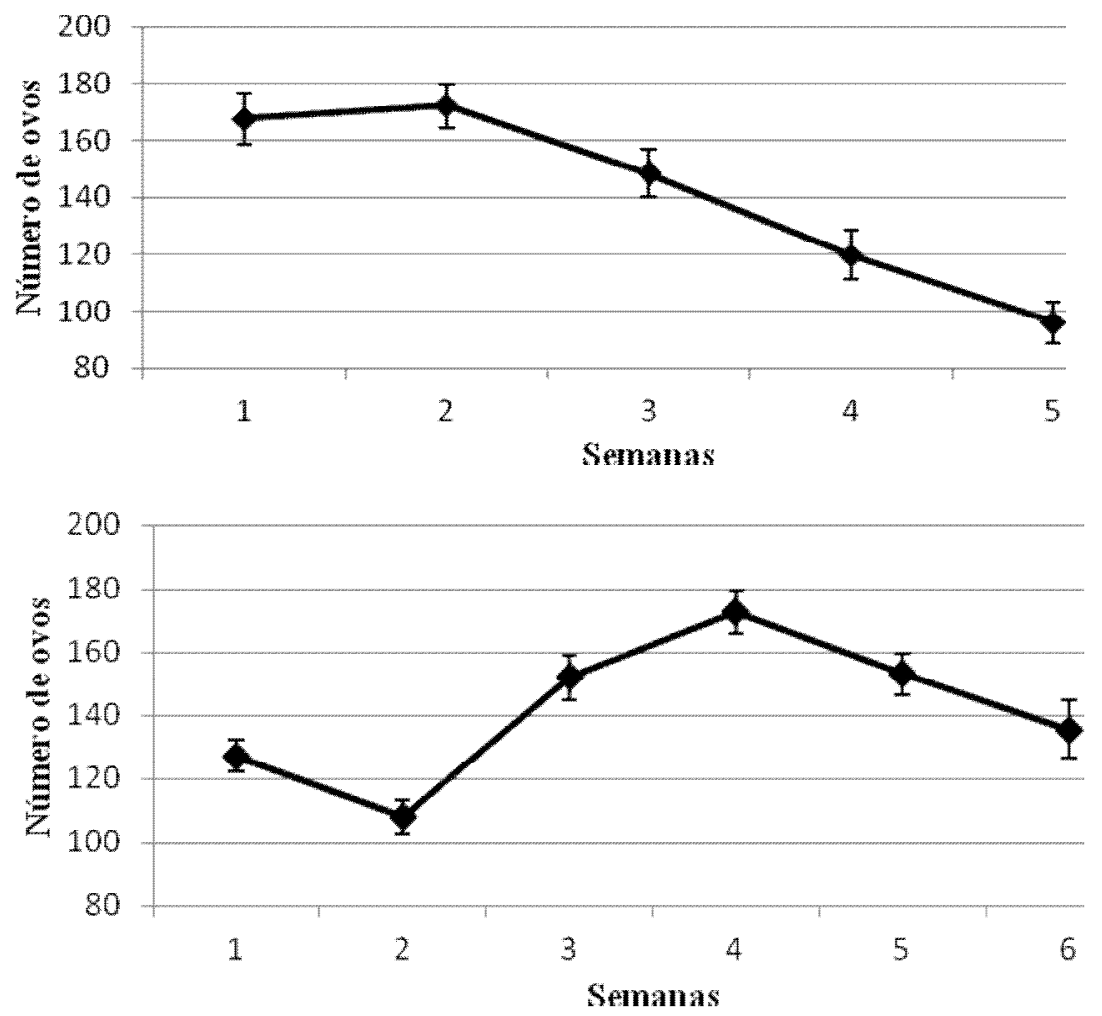

A viabilidade dos ovos (Tabela 1) ovipositados pelas fêmeas cujas fases imaturas foram submetidas a diferentes ambientes de criação parece não ter sido afetada por este fator abiótico, nem mesmo quando os ovos e larvas foram criados no ambiente em que houve flutuação da temperatura. Para este parâmetro biológico não foi observada diferença significativa entre os tratamentos.

Os valores médios observados quanto à longevidade de machos e fêmeas (Tabela 1) não apresentaram alteração significativa entre os tratamentos. Entretanto, as fêmeas avaliadas nestes experimentos viveram por mais tempo do que os machos, fato que corrobora os resultados obtidos pelos trabalhos de Núñez (1988) e Bezerra et al. (2006) em que ambos os autores observaram que as fêmeas sobreviveram por mais tempo.

Conhecer se a capacidade reprodutiva dos adultos de C. externa é afetada pelo fato de suas formas jovens terem sido submetidas a ambientes com diferentes temperaturas de criação é de grande importância para biofábricas. Desta forma, as biofábricas podem oscilar a temperatura de criação da melhor forma possível visando sincronizar sua produção com a necessidade de liberações em campo e assim, obter adultos com capacidade reprodutiva similar, não afetando a obtenção de ovos para as liberações.

\section{CONCLUSÃO}

A partir dos resultados obtidos é possível concluir que:

- As diferentes temperaturas de criação das fases imaturas de C. externa não influenciam os períodos de oviposição e pós-oviposição, o número de ovos total e diário, a viabilidade dos ovos e a longevidade de machos e fêmeas.

- Independente da temperatura de criação das fases imaturas de C. externa os adultos obtidos apresentam capacidade reprodutiva similar, não afetando a produção de ovos. Fato que é substancial para biofábricas que oscilam a temperatura para sincronia da produção de acordo com a demanda no campo. 


\section{AGRADECIMENTOS}

À FAPESP pela concessão de bolsa de iniciação científica (processo n ${ }^{\circ}$ 06/54392-3) durante o período de agosto de 2006 a julho de 2007.

\section{REFERÊNCIAS}

ALBUQUERQUE, G.S.; TAUBER, C.A.; TAUBER, M.J. Chrysoperla externa (Neuroptera: Chrysopidae): life history and potencial for biological control in Central and South America. Biological Control, v.4, p.8-13, 1994.

ANDREWARTHA, H.G.; BIRCH, L.C. Weather: Temperature. In: ANDREWARTHA, H.G.; BIRCH, L.C. (Eds). The Distribution and Abundance of Animals. London: The University of Chicago Press, 1954. p.129-171.

BEZERRA, G.C.D.et al. Aspectos biológicos da fase adulta de Chrysoperla externa (Hagen, 1861) (Neuroptera: Chrysopidae) oriunda de larvas alimentadas com Planococcus citri (Risso, 1813) (Hemiptera: Pseudococcidae). Ciência e Agrotecnologia, v.30, p.603-610, 2006.

CANARD, M.; VOLKOVICH, T.A. Outlines of lacewings development. In: MCEWEN, P.; NEW, T.R.; WHITTINGTON, A.E. (Eds.). Lacewings in the crop environment. Cambridge: Cambridge University Press, 2001. p.130-153.

ESTAT: sistema para análises estatísticas. Versão 2. Jaboticabal: FCAV, 1992.

MACEDO, L.P.M.et al. Efeito da idade das fêmeas e de fatores ambientais sobre a reprodução do predador Chrysoperla externa (Hagen, 1861) (Neuroptera: Chrysopidae). Acta Scientiarum: Biological Sciences, v.25, p.309-313, 2003b.

MAIA, W.J.M.S.et al. Capacidade predatória e aspectos biológicos de Chrysoperla externa (Hagen, 1861) (Neuroptera: Chrysopidae) alimentada com Rhopalosiphum maidis (Fitch, 1856) (Hemiptera: Aphididae). Ciência e Agrotecnologia, v.28, p.1259-1268, 2004.

NÚÑEZ, E. Ciclo biológico y crianza de Chrysoperla externa y Ceraeochrysa cincta (Neuroptera: Chrysopidae). Revista Peruana de Entomologia, v.31, p.76-82, 1988.

RIBEIRO, M.J.; CARVALHO, C.F. Aspectos biológicos de Chrysoperla externa (Hagen, 1861) (Neuroptera: Chrysopidae) em diferentes condições de acasalamento. Revista Brasileira de Entomologia, v.35, p.423-427, 1991. 